This is the peer reviewed version of the following article: Chen, Z., Zhao, C., Ju, E., Ji, H., Ren, J., Binks, B. P. and Qu, X. (2016), Design of Surface-Active Artificial Enzyme Particles to Stabilize Pickering Emulsions for HighPerformance Biphasic Biocatalysis. Adv. Mater., 28: 1682-1688, which has been published in final form at doi:10.1002/adma.201504557. This article may be used for non-commercial purposes in accordance With Wiley Terms and Conditions for self-archiving.

\title{
Design of Surface-active Artificial Enzyme Particles to Stabilize Pickering Emulsions for High-Performance Biphasic Biocatalysis
}

Zhaowei Chen, Chuanqi Zhao, EnguoJu, HaiweiJi, JinsongRen*, Bernard P. Binks*, and XiaogangQu*

Z. Chen, C. Zhao, E. Ju,H. Ji,Prof. Dr. J. Ren and Prof. Dr. X. Qu

Laboratory of Chemical Biology and State Key Laboratory of Rare Earth Resource

Utilization,Graduate School of the Chinese Academy of Sciences, ChangchunInstitute of

Applied Chemistry, Chinese Academy of Sciences, Changchun, Jilin 130022 (China)

E-mail: jren@ciac.ac.cn; xqu@ciac.ac.cn

Prof. B. P. Binks

Surfactant and Colloid Group, Department of Chemistry

University of Hull, Hull HU6 7RX (United Kingdom)

E-mail: b.p.binks@hull.ac.uk

Keywords: Pickering emulsions, artificial enzyme, biphasic biocatalysis, stability, re-usability

Enzymes represent as effective and sometimes preferable catalysts for a wide range of important organic reactions on account of their high chemo-, regio- and enantioselectivity. ${ }^{[1]}$ However, in many cases, substrates of interest are sparingly soluble in water whereas enzymes are typically active in the water phase. Thus aqueous-organic biphasic reaction systems are often employed to achieve high substrate concentration and enhanced productivity. Very recently, Pickering emulsions, which are emulsions stabilized by colloidal particles,${ }^{[2]}$ have emerged as a novel biphasic system for improving enzyme activity. ${ }^{[3]}$ Using Pickering emulsions not only facilitates mass transfer between the two liquid phases but also 


\section{WILEY-VCH}

simplifies product separation, as colloidal particles are easily recoverable. As a further evolution, Pickering interfacial biocatalysis where enzyme immobilized particles are employed as a whole to stabilize emulsions has become a more appealing proposition. ${ }^{[4]}$ In such a case the location of an enzyme at Pickering interfaces maximizes the oil-enzyme-water interfacial areas and increases the availability of the enzyme for substrates from both phases. Although promising, due to issues of low stability and environmental sensitivity, the application of enzymes for Pickering emulsion-based biocatalysis is still challenging. In particular, enzyme immobilization process and prolonged exposure to aqueous-organic interfaces easily result in changing enzyme natural structures, alternating enzyme activity and restricting re-usability. ${ }^{[3,4]}$

On the other hand, inspired by the structural complexity and the mechanistic pathways of natural enzymes, superstructures that incorporate typical enzyme catalytic centergroups (e.g. amino acid residues) and man-made elements into protein-like scaffolds have been studied in detail to develop artificial enzymes. ${ }^{[5]}$ For instance, multivalent nanostructures such as monolayer catalytic groups protected gold nanoparticles haveappeared as attractive biomimetic catalysts that follow principles of cooperativity and allostery, akin to natural enzymes. ${ }^{[6]}$ An intriguing property of artificial enzymes is their higher stability against denaturation under extreme conditions such as high temperature and even pure organic media. ${ }^{[7]}$ Also notably, the high catalytic activity, relative low-cost and easy preparation 


\section{WILEY-VCH}

features have prompted a myriad of novel developments in biomedical and synthetic applications. ${ }^{[8]}$

We envision that the unique merits of enzyme mimics would provide unprecedented opportunities for catalyzing biphasic reactions at Pickering emulsion droplet interfaces, overcoming the limits of natural enzymes. Beyond this, the unique physicochemical environment of Pickering emulsions is also able to provide a new platform to modulate the activity of artificial enzymes and even help elucidate factors influencing enzymatic processes under biphasic conditions. However, to the best of our knowledge, the construction of artificial enzymes for catalyzing bioconversions in Pickering emulsions has not been achieved.

Herein, surface-active artificial enzyme (SAE) particles were designed and constructed as a new generation of interfacial biocatalysts for oil-in-water $(\mathrm{o} / \mathrm{w})$ Pickering emulsions and their ability for high-performance biphasic biocatalysis is demonstrated. As shown in Scheme 1, by positionally locating the wettability-tuning groups and catalytic locus over and within porous colloidal particles, SAE capable of simultaneously stabilizing Pickering emulsions and catalyzing biphasic reactions could be obtained. Up to three types of SAE were synthesized by changing the catalytic groups from peptides to metal complexes to DNAzymes with a view to achieving esterase-, phosphotriesterase- and peroxidase-mimetic activities respectively. Notably, the assembly of the SAE at emulsion droplet interfaces dramatically enhanced the catalytic activity due to the greatly enlarged oil-water interfacial area and favored entry/egress 


\section{WILEY-VCH}

of substrates and products. Moreover, compared to their natural analogues, the SAE exhibited significantly higher operational stability against long-time exposure to organic solvents, as highlighted by the impressive re-usability for more than 20 cycles and high storage stability over 30 days with negligible loss of catalytic activity.

To test the validity of our design, surface-active artificial enzyme particles with esteraselike activity (ESAE) were fabricated first. Dendrimer-like mesoporous silica nanoparticles (DMSNs) with octyl group-modified outer surface and amino and thiol group-co-modified inner surface were prepared for hosting catalytic groups covered AuNP. ${ }^{13} \mathrm{C}-\mathrm{NMR}$ and ${ }^{29} \mathrm{Si}$ solid-state NMR spectra (Figure 1a, b) indicated the presence of the functional groups $\left(\mathrm{C}_{8}\right.$, $\mathrm{NH}_{2}$ and $\mathrm{SH}$ ). Scanning electron microscopy, SEM (Figure 1c and Figure S1) and transmission electron microscopy, TEM (Figure 1d) images showed the particle diameter of about $90 \mathrm{~nm}$ with central-radial pore size around $12 \mathrm{~nm}$. The mesoporosity was also validated by nitrogen adsorption measurements (Figure S2). Next, ultrafine AuNP of diameter 2-3 nm were prepared and attached to the inner surfaces of the pores via strong S-Au covalent bonds, as clearly shown in the TEM (Figure 1e, Figure S3), elemental mapping images (Figure 1f), UV-vis spectra (Figure S4) and X-ray photoelectron spectroscopy (XPS, Figure S5). Following this, catalytically active peptides (N-acetyl-Cys-His-Phe) were assembled onto the surfaces of the confined AuNPs. The Cys was used to make a covalent bond to the gold surface and the His-Phe was chosen because the carboxylate of Phe and the imidazole of His 


\section{WILEY-VCH}

act in a concerted way as general base and general acid at the active site of many esterasesduring the catalytic process. ${ }^{[6 a]}$ The variation in C1s XPS (Figure S5) and the appearance of an acylamide vibration band around $1656 \mathrm{~cm}^{-1}$ in the FTIR spectrum (Figure S6) confirmed the conjugation of the peptides. The amount of peptide anchored on the ESAE particles was about $0.092 \mathrm{mmol} \mathrm{g}^{-1}$ as quantified by elemental analysis (Table S1).

We next assessed the interfacial activity of the obtained ESAE particles for stabilizing Pickering emulsions. By homogenizing an equal volume $(1 \mathrm{~mL})$ of organic solvents such as dodecane, hexane, toluene, ethyl acetate and chloroform with water containing $0.6 \mathrm{wt} \%$ of ESAE particles, o/w Pickering emulsions were formed which remained stable to coalescence for at least one month (Figure 2a, Figure S7). The type of the formulated emulsions was further characterized under confocal laser scanning microscopy by dissolving fluorescein isothiocyanate labeled dextran (Dex-FITC) and Nile red in water and oil, respectively. With red emission in the droplet and green fluorescence in the continuous phase, o/w Pickering emulsions could be clearly identified (Figure 2b-e, Figure S8). The emulsion type was also verified by conductivity measurements (Figure S9). By contrast, no stable Pickering emulsion could be formed if the external surfaces of DMSN particles were not hydrophobized with organosilane, with the artificial enzyme being denoted $\operatorname{ESAE}_{(\text {no } \mathrm{C} 8)}$ (Figure 2a, right). A simple wettability study showed that the contact angle of a water drop in air on a layer of particles $(\theta$, Figure S10) was only around $17^{\circ}$ for $\operatorname{ESAE}_{(\text {no }}$ (8) particles, consistent with the inherent 


\section{WILEY-VCH}

hydrophilicity of the material, whereas it approached $72^{\circ}$ for ESAE particles indicating higher surface hydrophobicity. Also, water-in-oil (w/o) emulsions could be easily obtained by increasing the hydrophobicity of ESAE particles (Figure S11). Hence, sufficient surface hydrophobization was essential for conferring surface activity to the artificial enzymes enabling them to locate at emulsion droplet interfaces. SEM and TEM were further employed to scrutinize the microstructures of the ESAE-stabilized Pickering emulsions. For easy characterization, hydrophobic monomer, cross-linker and initiator were substituted as the oil phase prior to emulsification. After homogenization and polymerization, SEM (Figure 2f) and TEM images (Figure S12) clearly revealed that closely packed ESAE particles were observed on the surface of the polymer beads. Moreover, by embedding the beads in resin and cutting them into microtomed slices, ESAE particles were clearly seen only at the periphery of the beads with high-resolution SEM (Figure 2g). These results provided direct evidence for the pronounced interfacial activity of the obtained ESAE particles in Pickering emulsions. Such location of ESAE particles would favor the substrate from both phases to approach the biomimetic catalysts more easily.

The catalytic activity of the obtained ESAE particles at oil-water interfaces was studied with the hydrolysis of oil-soluble p-nitrophenyl butyrate (PNPB) using dodecane as the organic medium (Figure 3a). For benchmarking, the emulsions stabilized by particles with no peptide anchored on the AuNP (denoted as $\operatorname{ESAE}_{\text {(no peptide) }}$ ) served as background (control 1), 


\section{WILEY-VCH}

and neat biphasic water-dodecane mixtures containing monomeric peptides or non-surface active $\operatorname{ESAE}_{(\mathrm{no}} \mathrm{C8)}$ particles were used as control 2 and control 3, respectively. As shown in Figure $3 \mathrm{~b}$, the color of the aqueous phase became yellow as the reaction proceeded, indicating the catalytic activity of ESAE particles towards the hydrolysis of PNPB and phase transfer of the water-soluble product $p$-nitrophenolate anion (PNP, Figure S13). The kinetic study (Figure 3c) showed that ESAE particle-stabilized emulsions gave rise to rate accelerations at least 3 orders of magnitude relative to the background reaction and the second-order rate constant increased as a function of the amount of the anchored peptides on the particles (Figure S14), verifying that the peptides indeed contributed to the catalytic loci of the ESAE particles. Although minor, the activation in control 3 was significant since almost no measurable activity was observed in control 2, underpinning the importance of the confinement of peptides on AuNP surfaces for triggering the cooperative mechanism. ${ }^{[6 a]}$ Thus, by rationally locating different functional groups on particles, SAE with esterase-mimic activity could be successfully designed to stabilizing Pickering emulsions as well as catalyzing biphasic reactions.

Furthermore, the time course of the catalytic reaction shown in Figure S15 showed that the conversion of ESAE particle-catalyzed hydrolysis reached $96 \%$ at equilibrium, while much lower conversion was observed for the non-surface active $\operatorname{ESAE}_{(\text {no }}$ 8) particles in the neat biphasic system. The high catalytic activity of ESAE particles could be due to the 


\section{WILEY-VCH}

dramatically enlarged oil-water interfacial area within emulsions, which improved mass transfer and contact of ESAE particles with substrates from both phases. Evidence for this hypothesis was supported by studying the initial reaction rate of ESAE particle-stabilized emulsions with different emulsion droplet size. As anticipated, the reaction rate increased from 0.022 to $0.044 \mu \mathrm{M} \mathrm{s}^{-1}$ (Figure S16) while the mean droplet diameter decreased from $\sim 240$ to $\sim 70 \mu \mathrm{m}$ (Figure S17). Moreover, by varying the substrate concentration, the kinetics of the reaction within ESAE particle-stabilised emulsions was established to follow Michaelis-Menten theory (Figure S18). These results demonstrate that by changing the system parameters involved with Pickering emulsions, the system in turn can effectively modulate artificial enzyme activity, which is advantageous for optimizing factors determining the success of enzymatic processes.

Although ease of recovery of biocatalysts and particle emulsifiers is one of the apparent advantages of Pickering emulsions, the key factor for recycling biocatalysts is their long-term stability. This prompted us to investigate the re-usability of the ESAE particles for interfacial biocatalysis in Pickering emulsions. At the end of each catalytic reaction, the ESAE were recycled simply by centrifugation, washing and preparation for the next batch. Impressively, the results revealed very good recyclability with $90 \%$ of conversion efficiency maintained even after 20 cycles (Figure 4a). Conversely, in control experiments where natural esterase immobilized DMSN particles were used as emulsifier, the conversion efficiency declined 


\section{WILEY-VCH}

markedly with increasing cycles. ICP-MS and elemental analysis showed that almost no loss of $\mathrm{Au}$ and peptide was observed after 20 reaction cycles (Table S1), confirming the high stability of the ESAE particles at organic-aqueous interfaces. The inferior re-usability of natural enzymes could be attributed to the loss of natural structures during the immobilization and the prolonged exposure to organic-aqueous interface processes. Even worse, serious enzyme leaching was observed for the physically loaded esterase (Figure S19). Moreover, we found that the ESAE particles possessed higher storage stability compared with that of native esterase at emulsion droplet interfaces (Figure 4b). The residual activity of the ESAE particles after 30 days remained over $95 \%$ while the native esterase was less than $10 \%$. These results confirmed the superiority in terms of operational stability of utilizing synthetic artificial enzymes other than their natural analogues for prolonged exposure to Pickering emulsion interfaces.

To demonstrate the generality of our design, we also used a $\mathrm{Ce}^{\mathrm{IV}}$ complex as the catalytic centre $^{[9]}$ to construct phosphotriesterase-mimic SAE particles (PPSAE) for hydrolyzing organophosphonate (Figure 5, Figures S20-23). Many organophosphorous compounds are used as pesticides, insecticides and chemical warfare agents (CWA), all of which are toxic to mankind, and their detoxification is essential. ${ }^{[10]}$ Less toxic stimulants like $p$ nitrophenyldiphenyl phosphate (PNPDPP) was used to probe the activity of the PPSAE particles. It was notable that PPSAE particle-stabilized emulsions showed $85 \%$ of PNPDPP 


\section{WILEY-VCH}

conversion over the course of $60 \mathrm{~min}$ with a half-life of $21 \mathrm{~min}$. In addition, decreasing the emulsion droplet size increased the activity of PPSAE particles (Figure S24), implying the facilitated mass transfer and contact of PPSAE particles with reactants from the enhanced oilwater interfacial area. Finally, PPSAE particles are completely recyclable and possessed longterm storage stability (Figure S25, Table S2). Besides the $\mathrm{Ce}^{\mathrm{IV}}$ complex, a myriad of metal complexes could be employed to construct SAE as efficient metalloenzyme alternatives for biphasic biotransformation.

We also extended the methodology for its ability to construct SAE with catalytically active DNA (also known as DNAzymes). ${ }^{[11]}$ The G-quadruplex/hemin structure was used to construct SAE with peroxidase-like activity (POSAE, Figure 6, Figure S26 and S27). By using the catalytic reaction of $\mathrm{H}_{2} \mathrm{O}_{2}$ oxidation of hydrophobic (2-chloroethyl)ethyl sulfide (CEES, an organosulfur CWA simulant) as a model reaction, a remarkable decontamination capability was observed using POSAE particles with a half-life of $15.5 \mathrm{~min}$ (Figure 6c). An increase in the oil-water interfacial area within Pickering emulsions improved the detoxification efficiency without the need to increase the amount of peroxide (Figure S28). Also interestingly, GC-MS analysis indicated that the main product of the POSAE particlecatalyzed reaction was the relatively non-toxic sulfoxide (Figure 6d-e), which might be induced by a 2-e process involving the ferryl oxygen transfer to the $\mathrm{S}$ atom. ${ }^{[12]}$ Moreover, compared to native horseradish peroxidase, POSAE particles possessed better re-usability and 


\section{WILEY-VCH}

higher storage stability (Figure S29, Table S3). The results expand the properties and versatility of DNA-based artificial enzymes to more than just a pure aqueous environment. ${ }^{[13]}$

In summary, we have demonstrated the first example of the fabrication surface-active artificial enzymes (SAE) as a new generation of interfacial biocatalysts for high-performance biocatalysis in Pickering emulsions. By using peptide molecules, metal complexes and DNAzymes as the catalytic units, it was easy to build a diversity of SAE with esterase-, phosphotriesterase- and peroxidase-like activities for rapid bioprocessing of hydrophobic reactants. Significantly, we proved that the location of SAE particles at Pickering emulsion droplet interfaces greatly enhanced their catalytic activity due to the maximized oil-water interfacial area and facilitated mass transfer. Most importantly, compared to native proteins, SAE behaved as robust biocatalysts that withstand prolonged exposure to aqueous-organic interfaces with negligible loss of activity. Overall, the low cost, easy preparation, high stability and good re-usability of SAE offer promising features towards the design and construction of alternative types of interfacial biocatalysts in Pickering emulsions for potential applications in biotechnology, industrial synthesis and environmental protection.

\section{Supporting Information}

Supporting Information is available from the Wiley Online Library or from the author.

\section{Acknowledgements}




\section{WILEY-VCH}

This work was supported by National Basic Research Program of China (Grants 2012CB720602, 2011CB936004) and the National Natural Science Foundation of China (Grants 21210002, 21431007, 21202158, 21572216, 21533008).

Received: ((will be filled in by the editorial staff))

Revised: ((will be filled in by the editorial staff))

Published online: ((will be filled in by the editorial staff))

[1] A. M. Klibanov, Nature 2001, 409, 241; b) G. Carrea, S. Riva, Angew. Chem. 2000, 112, 2312;Angew. Chem. Int. Ed. 2000, 39, 2226.

[2] a) S. Crossley, J. Faria, M. Shen, D. E. Resasco, Science 2010, 327, 68; b) P. A. Zapata, J. Faria, M. P. Ruiz, R. E. Jentoft, D. E. Resasco, J. Am. Chem. Soc.2012, 134, 8570;

c) Z. Chen, H. Ji, C. Zhao, E. Ju, J. Ren, X. Qu, Angew. Chem. 2015, 127, 4986; Angew. Chem. Int. Ed. 2015, 54, 4904; d) Z. Chen, L. Zhou, W. Bing, Z. Zhang, Z. Li, J. Ren, X. Qu, J. Am. Chem. Soc. 2014, 136, 7498; e) I. Kosif, M. Cui, T. P. Russell, T. Emrick, Angew. Chem. 2013, 125, 6752; Angew. Chem. Int. Ed. 2013, 52, 6620;f) H. Yang, T. Zhou, W. Zhang, Angew. Chem. 2013, 125, 7603; Angew. Chem. Int. Ed. 2013, 52, 7455; g) W.-J. Zhou, L. Fang, Z. Fan, B. Albela, L. Bonneviot, F. De Campo, M. Pera-Titus, J.-M. Clacens, J. Am. Chem. Soc.2014, 136, 4869; h) B. P. Binks, J. A. Rodrigues, Angew. Chem. 2005, 117, 445; Angew. Chem. Int. Ed. 2005, 44, 441.

[3] a) C. Wu, S. Bai, M. B. Ansorge-Schumacher, D. Wang, Adv. Mater. 2011, 23, 5694;

b) Z. Wang, M. C. M. van Oers, F. P. J. T. Rutjes, J. C. M. van Hest, Angew. Chem. 2012, 124, 10904; Angew. Chem. Int. Ed. 2012, 51, 10746-10750; c) S. Wiese, A. C. Spiess, W. 


\section{WILEY-VCH}

Richtering, Angew. Chem. 2013, 125, 604; Angew. Chem. Int. Ed. 2013, 52, 576; d) D. C.

Dewey, C. A. Strulson, D. N. Cacace, P. C. Bevilacqua, C. D. Keating, Nat. Commun. 2014, 5, 4670; e) M. Li, R. L. Harbron, J. V. Weaver, B. P. Binks, S. Mann, Nat.Chem.2013, 5, 529.

[4] a) M. Pera-Titus, L. Leclercq, J.-M. Clacens, F. De Campo, V. Nardello-Rataj, Angew. Chem. 2015, 127, 2028; Angew. Chem. Int. Ed. 2015, 54, 2006; b) J. Liu, Y. Qihua, C. Li, G. Lan, J. Peng, Y. Li, Chem. Commun. 2013, 49, 9558; c) Y. Jiang, X. Liu, Y. Chen, L. Zhou, Y. He, L. Ma, J. Gao, Bioresource Technol. 2014, 153, 278.

[5] a) G. Y. Tonga, Y. Jeong, B. Duncan, T. Mizuhara, R. Mout, R. Das, S. T. Kim, Y.-C. Yeh, B. Yan, S. Hou, V. M. Rotello, Nat. Chem. 2015, 7, 597; b) KöhlerV, Y. M. Wilson, DürrenbergerM, GhislieriD, ChurakovaE, QuintoT, KnörrL, HäussingerD, HollmannF, N. J. Turner, T. R. Ward, Nat. Chem. 2013, 5, 93; c) V. Nanda, R. L. Koder, Nat.Chem. 2010, 2, 15.

[6] a) P. Pengo, S. Polizzi, L. Pasquato, P. Scrimin, J. Am. Chem. Soc. 2005, 127, 1616; b) M. Diez-Castellnou, F. Mancin, P. Scrimin, J. Am. Chem. Soc. 2014, 136, 1158; c) D. Zaramella, P. Scrimin, L. J. Prins, J. Am. Chem. Soc. 2012, 134, 8396; d) C. Zhang, T. Pan, C. Salesse, D. Zhang, L. Miao, L. Wang, Y. Gao, J. Xu, Z. Dong, Q. Luo, J. Liu, Angew.Chem. 2014, 126, 13754; Angew. Chem. Int. Ed. 2014, 53, 13536; e) A. A. Neverov, Z.-L. Lu, C. I. Maxwell, M. F. Mohamed, C. J. White, J. S. W. Tsang, R. S. Brown, J. Am. Chem. Soc. 2006, 128, 16398; f) Y. Lin, J. Ren, X. Qu, Accounts Chem.Res. 2014, 47, 1097. 


\section{WILEY-VCH}

[7] a) N. Kapil, A. Singh, D. Das, Angew. Chem. 2015, 127, 6592; Angew. Chem. Int. Ed. 2015, 54, 6492; b) Q. Wang, Z. Yang, X. Zhang, X. Xiao, C. K. Chang, B. Xu, Angew. Chem. 2007, 119, 4363; Angew. Chem. Int. Ed. 2007, 46, 4285.

[8] a) A. A. Vernekar, D. Sinha, S. Srivastava, P. U. Paramasivam, P. D’Silva, G. Mugesh, Nat. Commun. 2014, 5, 5301; b) F. Natalio, R. Andre, A. F. Hartog, B. Stoll, K. P. Jochum, R.

Wever, W. Tremel, Nat. Nano. 2012, 7, 530; c) L. Gao, J. Zhuang, L. Nie, J. Zhang, Y. Zhang, N. Gu, T. Wang, J. Feng, D. Yang, S. Perrett, X. Yan, Nat. Nano. 2007, 2, 577; d) B. Liu, Z. Sun, P.-J. J. Huang, J. Liu, J. Am. Chem. Soc. 2015, 137, 1290; e) K. Li, K. Wang, W. Qin, S. Deng, D. Li, J. Shi, Q. Huang, C. Fan, J. Am. Chem. Soc. 2015, 137, 4292; f) Y. Song, K. Qu, C. Zhao, J. Ren, X. Qu, Adv. Mater. 2010, 22, 2206; g) H. Sun, N. Gao, K. Dong, J. Ren, X. Qu, ACS Nano 2014, 8, 6202.

[9] R. Bonomi, P. Scrimin, F. Mancin, Org. Biomol. Chem.2010, 8, 2622-2626.

[10] J. E. Mondloch, M. J. Katz, W. C. Isley Iii, P. Ghosh, P. Liao, W. Bury, G. W. Wagner, M. G. Hall, J. B. DeCoste, G. W. Peterson, R. Q. Snurr, C. J. Cramer, J. T. Hupp, O. K. Farha, Nat. Mater. 2015, 14, 512.

[11] a) S. K. Silverman, Chem. Commun. 2008, 3467; b) C. Wang, G. Jia, J. Zhou, Y. Li, Y. Liu, S. Lu, C. Li, Angew. Chem. 2012, 124, 9486; Angew. Chem. Int. Ed. 2012, 51, 9352.

[12] E. Baciocchi, O. Lanzalunga, S. Malandrucco, M. Ioele, S. Steenken, J. Am. Chem. Soc.1996, 118, 8973. 


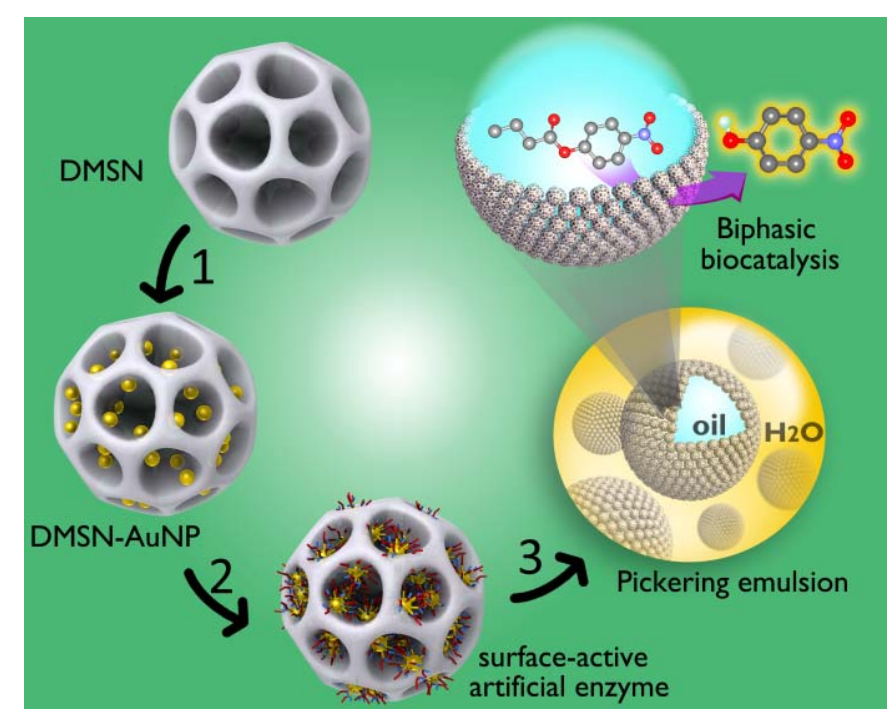

Scheme 1.General procedure for the construction of surface-active artificial enzymes (SAE) as interfacial biocatalysts in Pickering emulsions. (1) Firstly, dendrimer-like mesoporous silica nanoparticles (DMSN) with octyl group-modified outer surface,and aminosilane and thiolsilane co-modified inner surface were synthesized. Ultrafine gold nanoparticles (AuNP) were deposited on the inner surfaces of DMSN via Au-S bonds. (2) Then, one monolayer of thiol-ended catalytic groups is anchored on the surfaces of the AuNPs to form the catalytic loci. Up to three kinds of catalytic groups ranging from peptides ( $N$-acetyl-Cys-His-Phe) to $\mathrm{Ce}^{\mathrm{IV}}$-nitrilotriacetic acid complex to G-quadruplex/hemin were employed to construct SAE with esterase-, phosphotriesterase- and peroxidase-like activities, respectively. (3) Assembly of the SAE at Pickering emulsion droplet interfaces for biphasic biocatalysis. By loading hydrophobic substrates in the oil phase, the SAE can effectively catalyze the reactants in the water phase with the oil-soluble reactants, e.g. the conversion of $p$-nitrophenyl butyrate into water-soluble $p$-nitrophenolate catalyzed by esterase-mimic SAE. 


\section{WILEY-VCH}

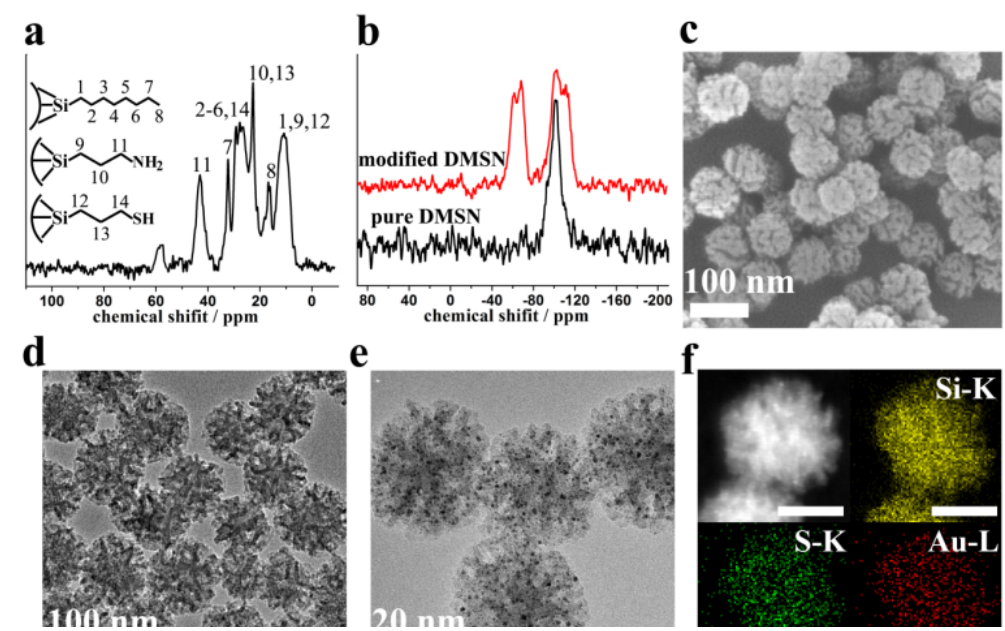

Figure 1.(a) ${ }^{13} \mathrm{C}$ solid-state NMR spectrum of the octyl-/amino/thiol-modified DMSN, chemical shift at $\delta=58 \mathrm{ppm}$ was from the oxidized 3-mercaptopropyl group). (b) ${ }^{29} \mathrm{Si}$ solidstate NMR spectrum of pure DMSN and modified DMSN (T $T^{2}:-61 \mathrm{ppm} ; \mathrm{T}^{3}:-68 \mathrm{ppm} ; \mathrm{Q}^{3}$ : $101 \mathrm{ppm} ; \mathrm{Q}^{4}$ : -111 ppm). (c) SEM and (d) TEM images of the DMSN-NH $/ 2 \mathrm{SH} / \mathrm{C} 8$ particles, showing the dendrimer-like mesoporous structure. (e) TEM image after depositing ultrafine AuNP in the pores of DMSN particles and (f) corresponding elemental mappings of the Si Kedge, S-K edge and Au L-edge siganls; in (f) scale bars: $50 \mathrm{~nm}$.
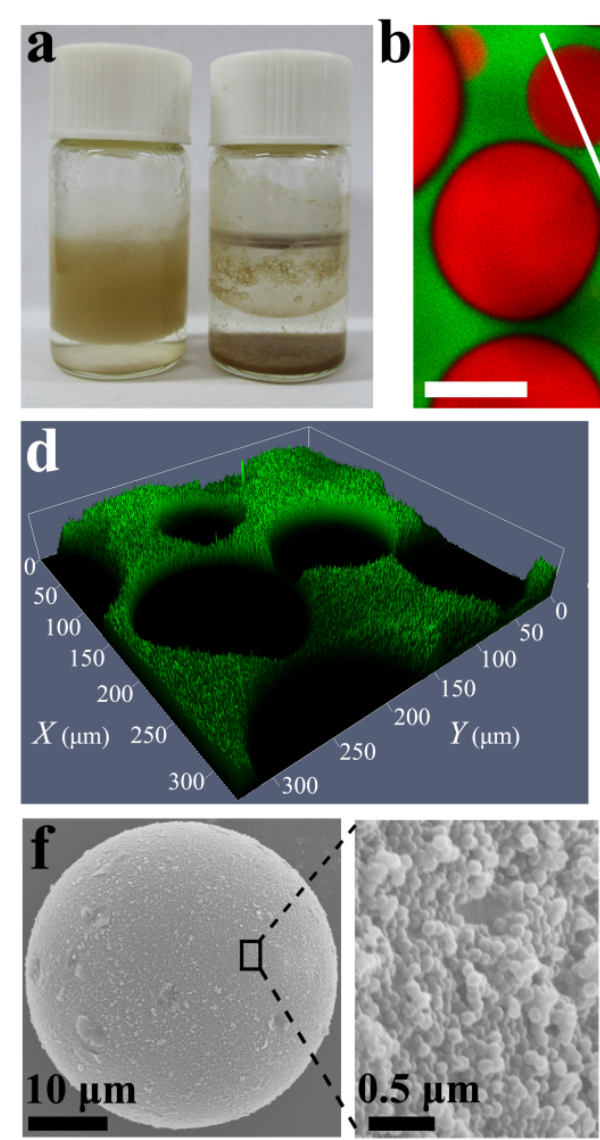
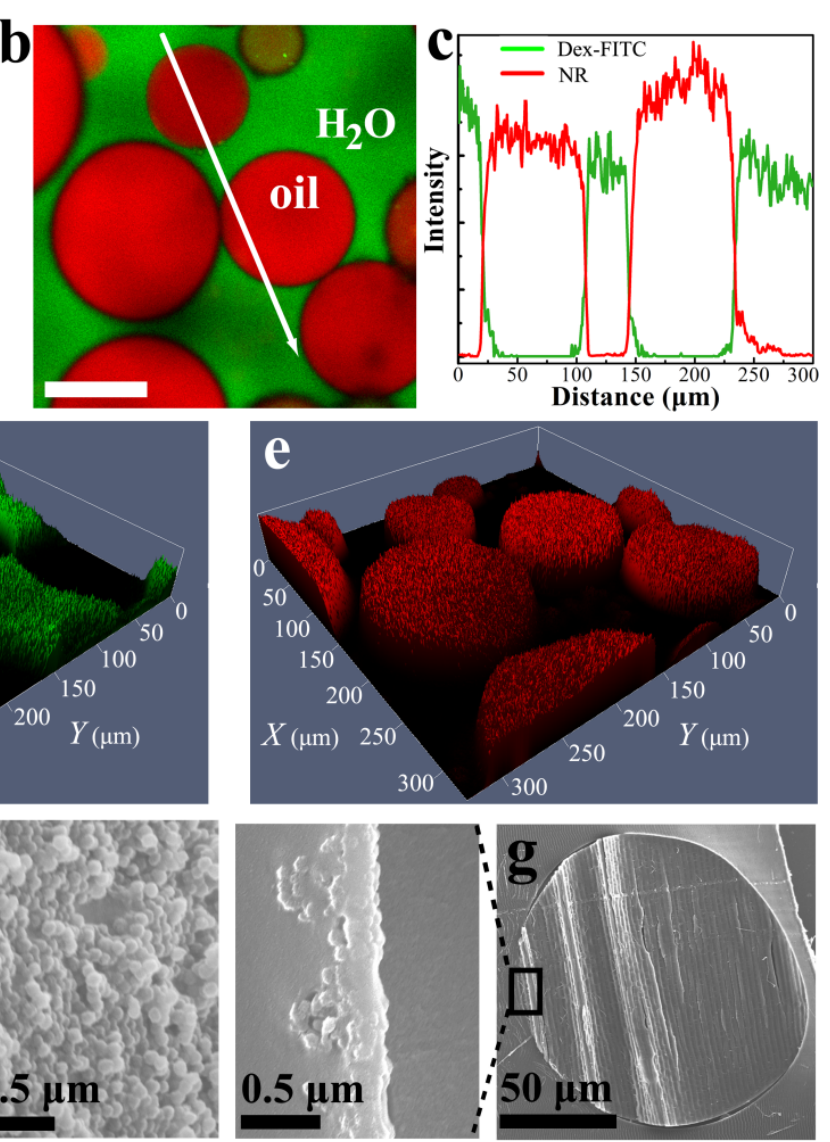

Figure 2.Interfacial activity of the obtained artificial enzymes within Pickering emulsions. (a) The appearance after $24 \mathrm{~h}$ of dodecane-in-water Pickering emulsions stabilized by ESAE 


\section{WILEY-VCH}

(left) and control non-surface active $\operatorname{ESAE}_{(\mathrm{noC} 8)}$ (right), indicating surface hydrophobization of DMSN particles was necessary to confer SAE surface-activity. (b) 2D confocal laser scanning microscopy (CLSM) of ESAE-stabilized o/w Pickering emulsion with Dex-FITC in water and Nile red in dodecane (scale bar: $100 \mu \mathrm{m}$ ). (c) Fluorescence intensity profile of the highlighted emulsion droplets shown in panel b. (d) and (e) 3D CLSM of the Pickering emulsions shown in the green and red channel, respectively. (f) SEM image of the polymerized Pickering emulsion droplets by loading monomer and initiator in the oil phase; the ESAE can be clearly seen located on the emulsion droplet surfaces in the magnified image. (g) SEM image of the microtomed slices of polymerized Pickering emulsion droplet showing the distribution of ESAE only at the periphery of the droplet.

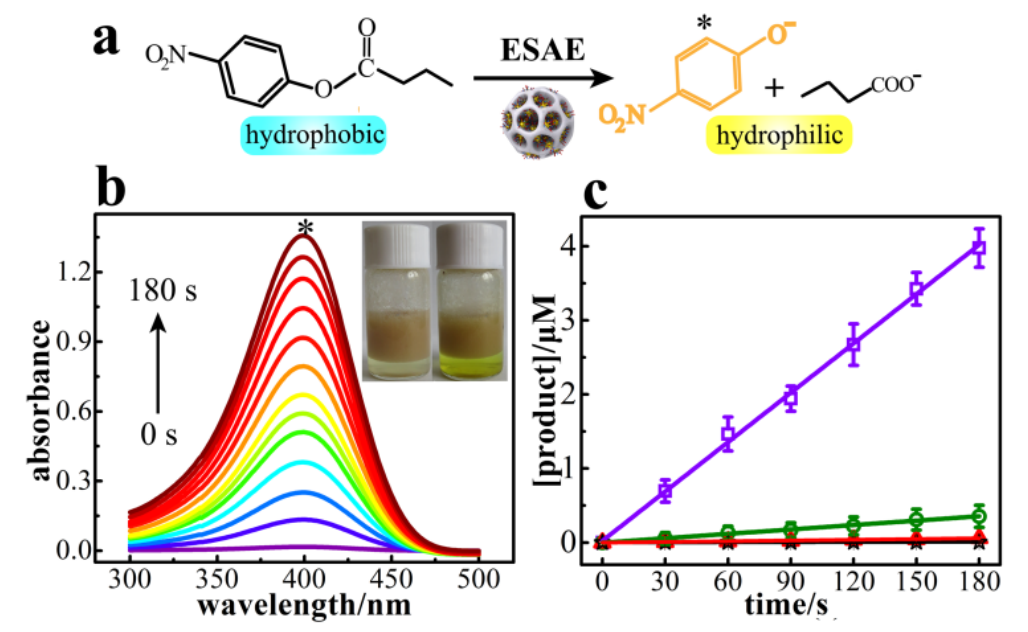

Figure 3.(a) ESAE-catalyzed conversion of oil-soluble PNPB into water-soluble PNP and butyric acid. (b) UV-visible monitoring of the formation of PNP and its transfer into the water phase. Inset: photograph showing the ESAE-catalyzed hydrolysis of PNPB and the phase-transfer of the product PNP into the water phase at $0.3 \mathrm{~min}$ (left) and $5 \mathrm{~min}$ (right) after the addition of PNPB in the oil phase. (c) The initial PNPB hydrolysis profile catalyzed by ESAE particles in o/w Pickering emulsions $(\square)$, emulsions stabilized by $\operatorname{ESAE}_{\text {(no peptide) }}$ particles ( $\downarrow$, control 1), monomeric peptide $(\Delta$, control 2$)$ and non-surface active $\operatorname{ESAE}_{\text {(no }} 8$ ) in neat biphasic system ( $\circ$, control 3$)$, respectively.
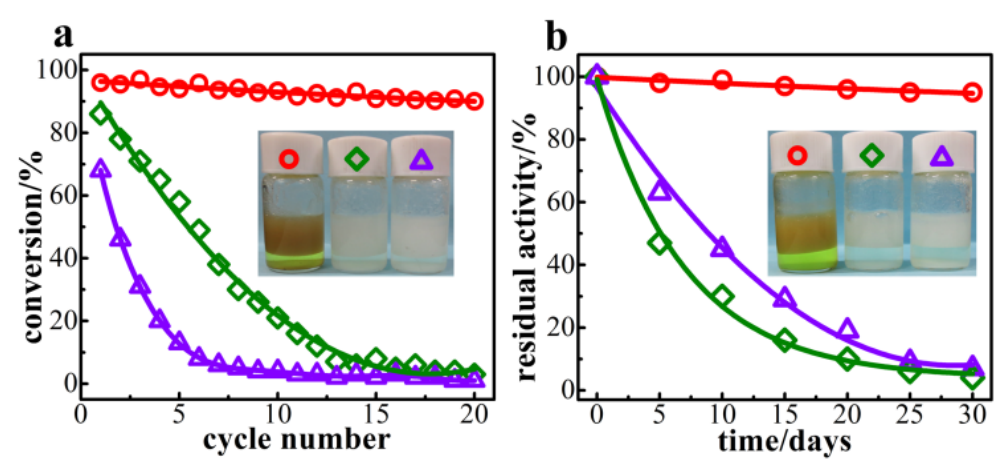


\section{WILEY-VCH}

Figure 4. (a) Recycling conversion efficiency of SAE particles ( $($ ), esterase covalently immobilized DMSN particles $(\diamond)$ and esterase physically loaded DMSN particles $(\Delta)$ as interfacial biocatalysts in Pickering emulsions. For the cases shown in panel a, (b) shows the retention of enzymatic activity at different times after locating at Pickering emulsion droplet interfaces $\left(25{ }^{\circ} \mathrm{C}\right)$. Each data point represents an average of triplicate measurements with standard error $<10 \%$. Inset photographs in (a) and (b) are for the catalytic activity after 20 cycles and 30 days, respectively. The photos were taken 5 min after addition of $200 \mu \mathrm{M}$ PNPB in the oil phase.
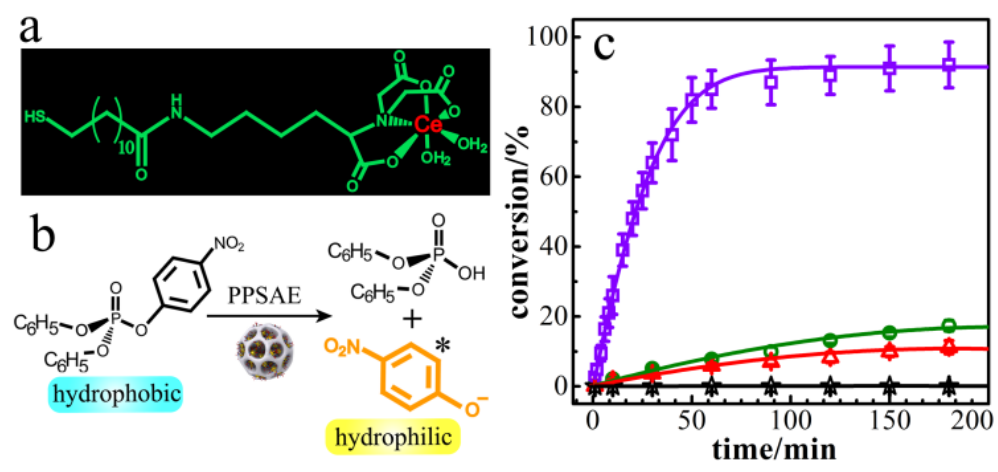

Figure 5.(a) Molecular structure of the $\mathrm{Ce}^{\mathrm{IV}}$ complex utilized to construct PPSAE. (b) PPSAE-catalyzed hydrolytic reaction for decontamination of oil-soluble PNPDPP into hydrophilic PNP. (c) The conversion course of PNPDPP versus time for PPSAE particlestabilized Pickering emulsions ( $\square$ ); background $\operatorname{PPSAE}_{(\text {no Ce) }}$ particle-stabilized Pickering emulsions ( $\langle$, control 1$)$; monomeric $\mathrm{Ce}^{\mathrm{IV}}$ complex $(\Delta$, control 2) or non-surface active $\left.\operatorname{PPSAE}_{(\mathrm{no}} \mathrm{C} 8\right)$ in neat biphasic system ( $\circ$, control 3$)$, respectively.
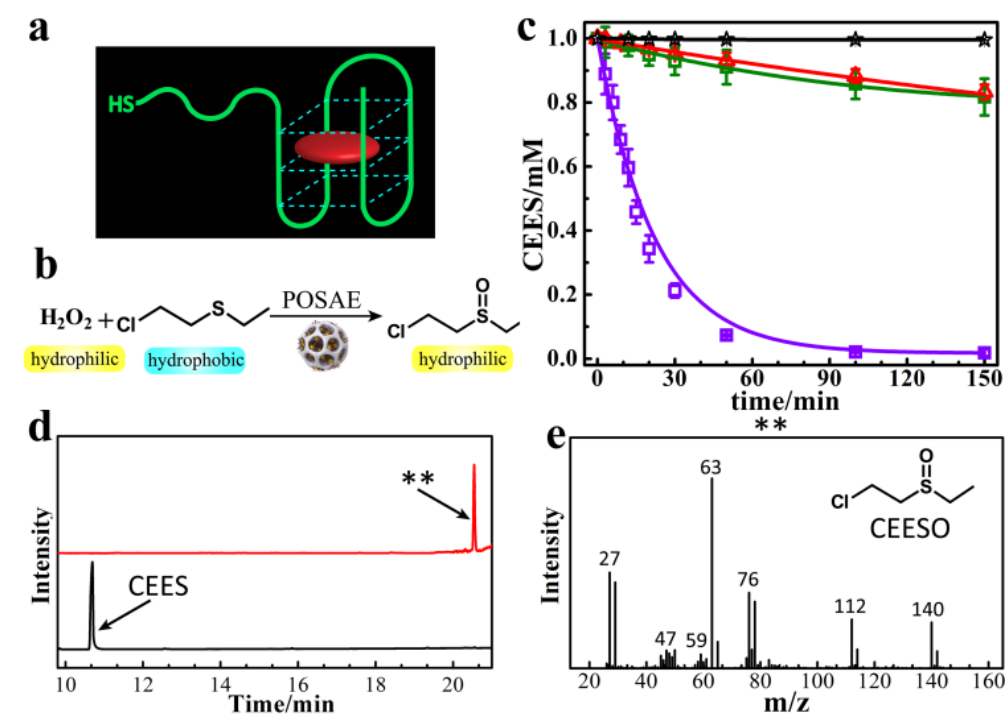

Figure 6.(a) Structure of G-quadruplex/hemin used to construct POSAE particles; the molecular structure of hemin is shown in Figure S26. (b) POSAE particle-catalyzed oxidation 


\section{WILEY-VCH}

of hydrophobic (2-chloroethyl)ethyl sulfide (CEES) with hydrophilic hydrogen peroxide. (c) The oxidative abatement of CEES with hydrogen peroxide for POSAE particles within

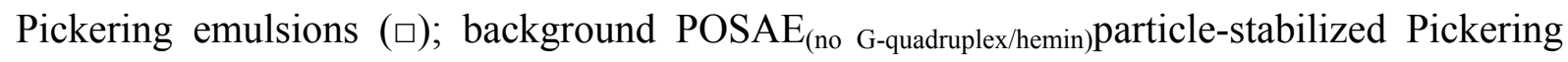
emulsions ( 论, control 1); monomeric G-quadruplex/hemin structures ( $\Delta$, control 2) or nonsurface active $\operatorname{POSAE}_{(\mathrm{no} C 8)}$ in neat biphasic system (O, control 3), respectively. (d) and (e) GC-MS analysis indicates the oxidation of CEES to CESSO ((2-chloroethyl)ethyl sulfoxide) using POSAE particles in Pickering emulsions. 


\section{WILEY-VCH}

\section{The table of contents entry}

KeywordPickering emulsions, artificial enzyme,biphasic biocatalysis,stability,re-usability

Zhaowei Chen, Chuanqi Zhao, EnguoJu, HaiweiJi, JinsongRen*, Bernard P. Binks*, and XiaogangQu*

TitleDesign of Surface-active Artificial Enzyme Particles to Stabilize Pickering Emulsions for High-Performance Biphasic Biocatalysis

A general and novel strategyispresent for design and construction of surface-active artificial enzymes (SAEs) that can simultaneously stabilize Pickering emulsions and catalyze biphasic biotransformationwith superior enzymatic stability and well re-usability; for example for the interfacial conversion of hydrophobic $p$-nitrophenyl butyrate into yellow water-soluble $p$ nitrophenolate catalyzed by esterase-mimic SAE.

ToC figure

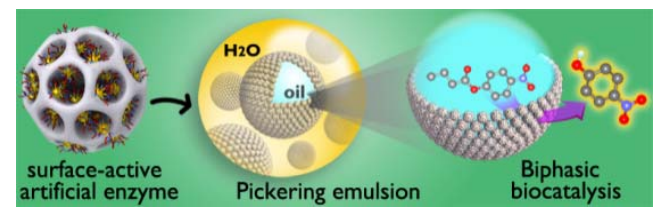

